\title{
Progressive dyspnea in patient with large mediastinal mass
}

\author{
Shinichi Fukuhara, Kamellia R Dimitrova, Charles M Geller, Darryl M Hoffman, Wilson Ko and Robert F Tranbaugh ${ }^{*}$
}

\begin{abstract}
Liposarcoma occurs very rarely in the mediastinum. Patients often remain asymptomatic until it grows large enough to cause direct invasion or compression of adjacent organs. We report a case of a 77-year-old male presented with dyspnea of exertion and was found to have a large mediastinal mass which was eventually diagnosed as primary mediastinal well-differentiated liposarcoma. The limited respiratory function at the initial presentation prompted phrenic nerve preserving incomplete resection rather than radical removal of the adjacent mediastinal structures. After surgical removal, the recurrence for well-differentiated mediastinal liposarcomas in the mediastinum is unknown; therefore, close follow-up is crucial.
\end{abstract}

Keywords: Primary mediastinal neoplasm, Liposarcoma, Surgical resection

\section{Background}

Liposarcoma is the most commonly diagnosed soft tissue sarcoma in adults but occurs very rarely in the mediastinum [1]. The tumors are often asymptomatic until large which causes symptoms from direct invasion or compression of the heart, great vessels or lungs [2]. Gross examination cannot distinguish between lipoma and liposarcoma. In the majority of cases, computed tomography $(\mathrm{CT})$ and/or magnetic resonance imaging (MRI) are sufficient to determine the nature of the tumor [3-6]. The behavior of any liposarcoma is dependent on its histological subtype. The treatment principles are the same as for other soft tissue sarcomas including surgical removal with adjuvant radiation and chemotherapy [7-9]. We herein describe a case of liposarcoma of mediastinum in a 77-year-old man.

\section{Case presentation}

A 77-year-old Hispanic male presented with a six months history of exertional dyspnea and recent onset of chest pain. He denied fevers or weight loss. His vital signs were normal. Physical examination demonstrated dullness on percussion and decreased breath sounds at the bases bilaterally. Forced expiratory volume in $1 \mathrm{sec}-$ ond (FEV1) was $1.78 \mathrm{~L}$ and forced vital capacity (FVC)

\footnotetext{
* Correspondence: RTranbau@chpnet.org

Division of Cardiac Surgery, Beth Israel Medical Center, 317 East 17th Street, 11th Floor, New York, NY 10003, USA
}

was $2.29 \mathrm{~L}$. Laboratory data, electrocardiogram and arterial blood gas analyses were normal. Chest radiograph demonstrated a widened mediastinum and bilateral lower lobe haziness (Figure 1). A chest CT showed a well-defined, lobulated, heterogenous mass of low attenuation in the anterior mediastinum. The mass abutted the ascending aorta, pulomonary artery, right hemidiaphragm, and the pericardium with focal obliteration of fat planes (Figure 2). Patient refused MRI, but the bone scan, head and abdominal CT scans showed no other lesions. The patient underwent median sternotomy and en block resection of the tumor. An extremely large, well-demarcated and multi-lobulated mass was resected superiorly along the trachea, laterally to both phrenic nerves and inferiorly to both hemi-diaphragms. Both phrenic nerves were preserved with minimal surrounding tissue. The pericardium was intact with no invasion of the heart or great vessels. The tumor measured $36 \times 20 \times 6.5 \mathrm{~cm}$ and weighed $2930 \mathrm{~g}$ (Figure 3). Macroscopically, the mass was soft and pale to dark yellow in color, well-circumscribed with rubbery nodules within the specimen. Histologically, the tissue was composed of variable sized mature lipocytes (Figure 4A) with large sharply outlined vaculoles (Lochkern vacuoles), fibromyxoid stroma containing spindle cells (Figure 4C) and lipoblasts with mitotic rate of 1 to 3 per 10 high-power fields (Figure 4B). Final pathological diagnosis was primary mediastinal well- 


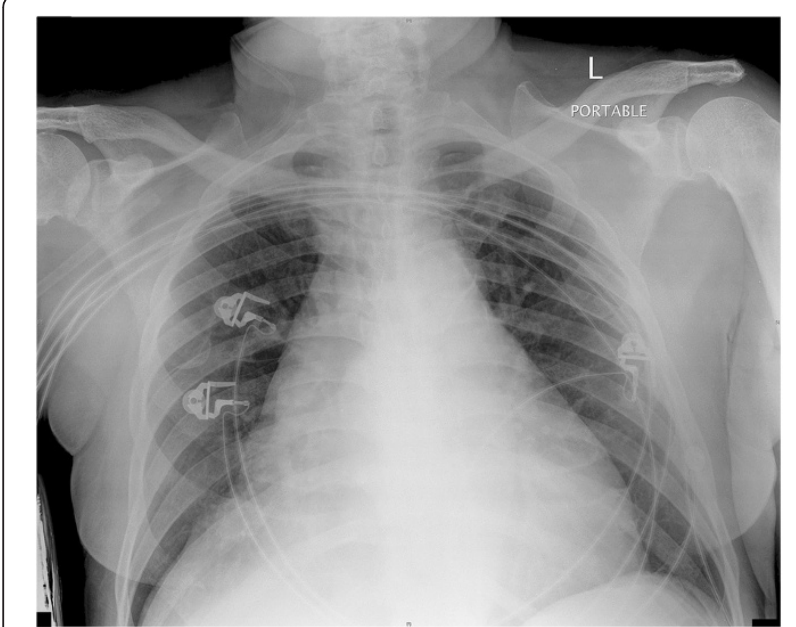

Figure 1 Preoperative antero-posterior chest radiograph.

Antero-posterior chest radiograph shows widened mediastinum and parenchymal haziness at the bases of both lungs.

differentiated liposarcoma without extra-mediastinal foci of tumor. Patient was discharged home 8 days after the surgery. Radiotherapy was recommended; however, our patient declined. He was asymptomatic two years after discharge and undergoes surveillance CT of the chest yearly.

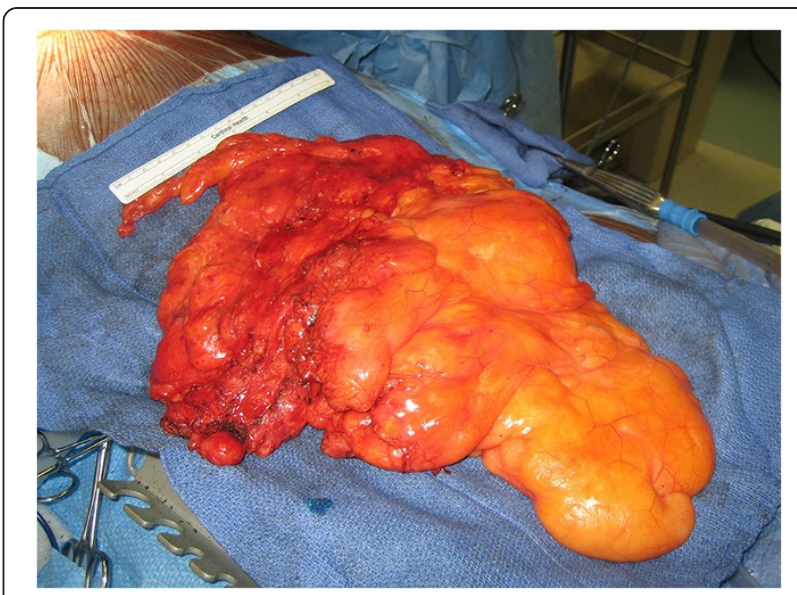

Figure 3 Intraoperative photograph. Intraoperative specimen, liposarcoma appearance is similar to that of mature fat.

\section{Discussion}

Liposarcoma most commonly occurs in the lower extremities and retroperitoneum and rarely in the subcutaneous tissue, paratesticular fat tissue and the mediastinum [1]. Most patients with liposarcomas present complaining of a large mass, often painless unless some sort of trauma has occurred or a vital organ is compressed [2]. Common presenting symptoms of mediastinal liposarcomas are chest pain, dyspnea, wheezing, cough and weight loss [10].

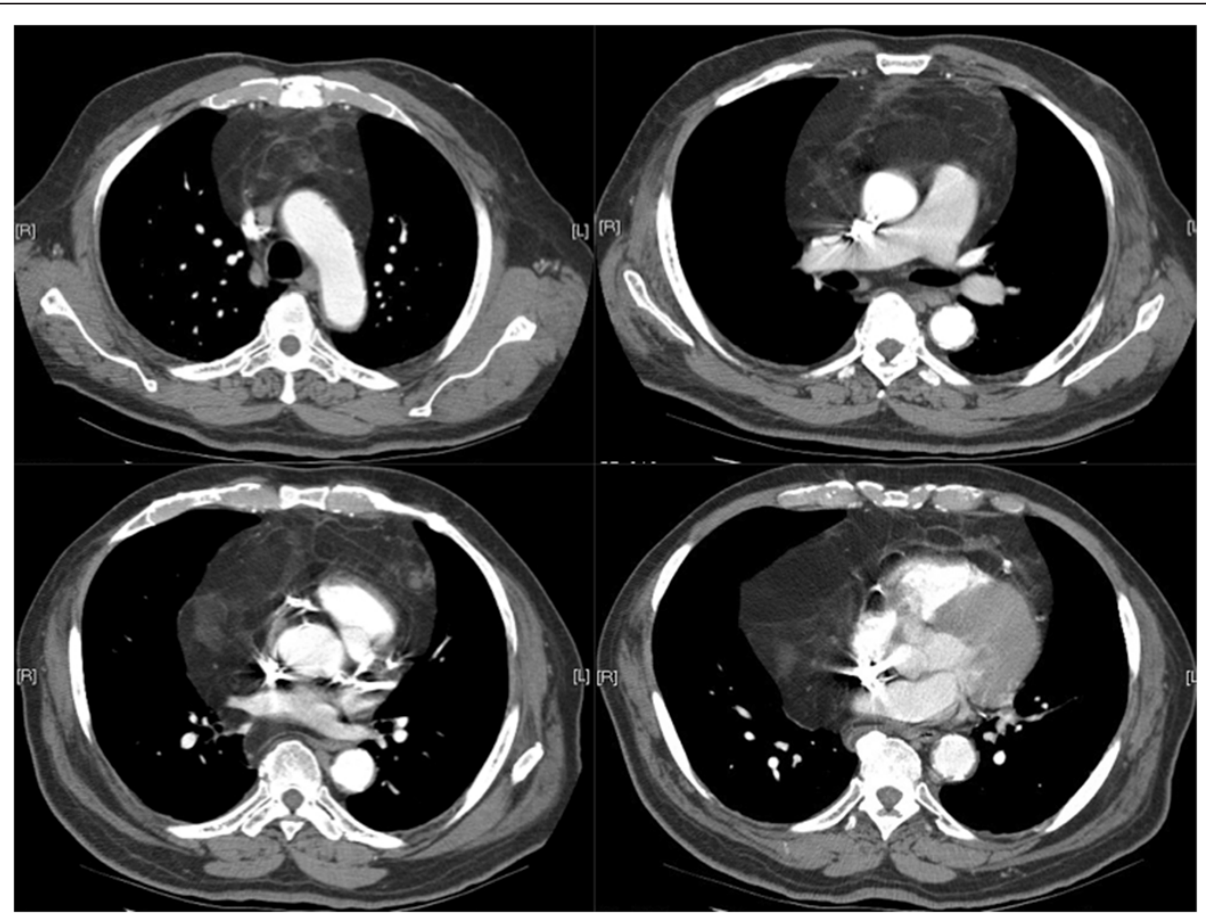

Figure 2 Preoperative computed tomography scan. Chest computed tomography scan at the level of pulmonary hilum (upper left image) demonstrates the multi-lobulated mass, well-defined, homogenous, low-attenuation mass in the anterior mediastinum. The other three images show lobulated mass with multiple calcific septations. The fat plane between the mass and the left pulmonary artery is focally obliterated. 


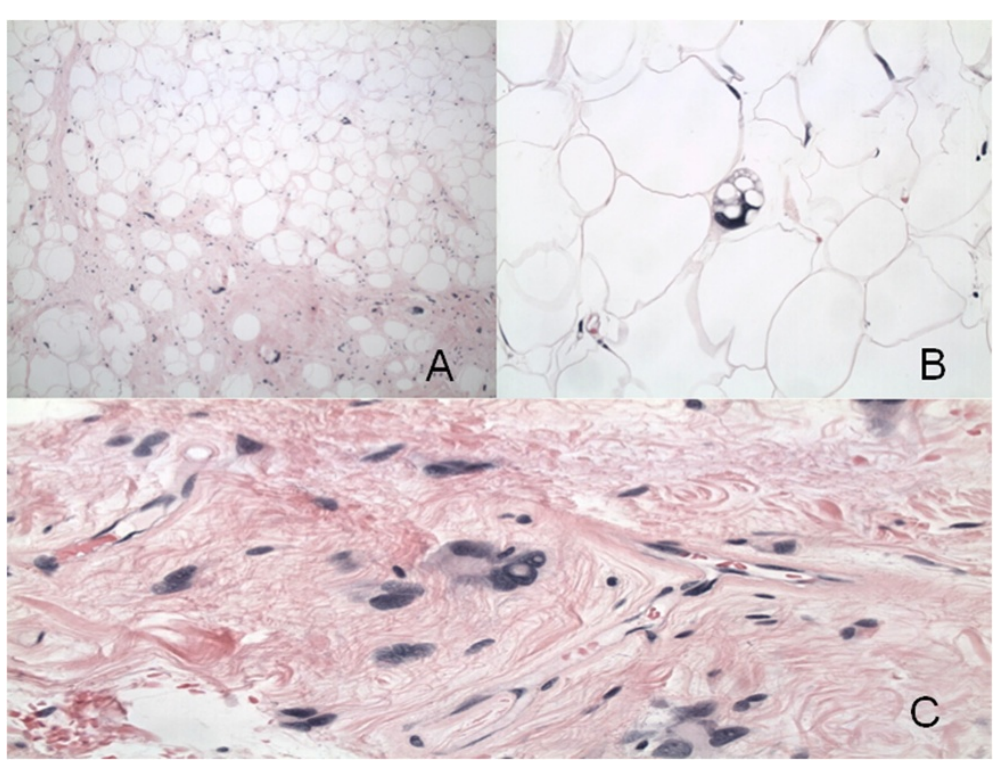

Figure 4 Histological sections. A, Adipose cells and fibrous strands with scattered "signet-ring" type cells resembling normal adipose tissue but with large, deep-stranding nuclei (hematoxylin-eosin, original magnification $\times 200$ ). B, Lipoblast showing lipid vacuoles and indented nucleus (hematoxylin-eosin, original magnification $\times 400$ ). C, Lochkern vacuole (hematoxylin-eosin, original magnification $\times 200$ ).

\section{Table 1 Liposarcoma subtypes}

\begin{tabular}{ll}
\hline Well-differentiated & Most common subtype (50\% of liposarcomas) \\
& Mature adipocytes with large fatty vacuoles \\
& None of a few lipoblasts \\
& Infrequent mitosis \\
& Variable myxomatous tissue with occasional \\
& dense hyaline sclerosis \\
& Occasional spindle cells \\
& Low grade with risk of dedifferentiation \\
& Most common type in pediatric age group \\
& Immature mesenchymal giant cells in prominent \\
& mucopolysaccharide rich stroma \\
& Lipoblasts with mitotic figures \\
& Prominent vascularization of branching capillaries \\
& (chicken wire pattern) \\
& Includes round-cell variant as its high-grade \\
& counterpart \\
& Intermediate grade with metastatic risk \\
& especially in round-cell variant \\
& Rarest type (5-10\% of liposarcomas) \\
& Many lipoblasts with high mitotic rate \\
& Highly anaplastic sarcoma \\
& High grade with high risk of local recurrence \\
& and metastasis \\
& May mimic carcinoma or melanoma \\
& Most common with retroperitoneal lesions \\
& High grade with very hight risk of metastasis \\
Dedifferentiated &
\end{tabular}

Pericardial and pleural effusions are common, although localized lung collapse is characteristic clinical sign when it reaches larger size [10]. Factors that suggest malignancy are masses greater than $5 \mathrm{~cm}$ and lesions that are deepseated, firm and fixed to underlying structures [1]. Mediastinal liposarcomas are more often found in the anterior mediastinum, adherent to the pericardium and tend to involve the diaphragm and phrenic nerves $[11,12]$. Esophagoscopy and bronchoscopy may reveal an extrinsic compression effect of the tumor, but no evidence of an intraluminal lesion. In cases where metastatic disease is suspected a transthoracic CT-guided core-needle biopsy or mediastinoscopy is indicated to obtain tissue specimen to establish the diagnosis [12].

Findings in plain chest radiography are nonspecific, and offer little except confirming the presence of a soft-tissue mass. CT provides valuable morphologic information and helps in the differentiation of various types of lipomatous tumors [1-6,10-12]. The typical liposarcoma appearance on CT and MRI is a heterogenic mass composed of adipose tissue mixed with non-adipose components. Radiographic features suggesting higher grade malignancies are large lesion size, presence of thick septations and nodular and/or globular non-adipose mass like areas, and decrease percentage of fat [6]. Well-differentiated liposarcomas are typically composed of more than $75 \%$ fat, while the other histologic types usually have less than $25 \%$ [6]. Liposarcomas are radiosensitive soft-tissue tumors and the more aggressive tumors demonstrate more 
radiopacity [5]. Poorly differentiated liposarcomas, particularly the pleomorphic type liposarcoma, may be indistinguishable from other mediastinal malignancies such as malignant fibrous histiocytoma, leiomyosarcoma, desmoids, mesothelial tumors, lymphomas, metastases, and inflammatory masses.

The pathological evaluation is the most important step to differentiate between mediastinal liposarcoma and all other mediastinal fat-containing tumors: lipoma, thymolipoma, teratoma, lymphoma, germ cell tumor, or even herniated peritoneal fat. The World Health Organization currently recognized four subtypes of liposarcoma: welldifferentiated, myxoid, pleomorphic and dedifferentiated (Table 1) [13]. Well-differentiated liposarcomas have histologic features resembling mature adipose tissue, as the presence of fibrous tissue septa and spindle cells is the main distinctive pathologic characteristic between them [14]. Liposarcomas are locally aggressive tumors composed of enlarged adipocytes ("signet-ring" type cells) with atypical hyperchromatic cells with angular nuclei and lipoblasts [15]. Overall the cellularity is low and mitotic figures are uncommon, with rare heterologous differentiation and none or only a few lipoblasts. Macroscopically well-differentiated liposarcomas also resemble lipomas, but tend to be larger, often traversed by dense bands of collagen, gelatinous areas, and nodularity with greater variation in size and firmness than an ordinary lipoma. Well-differentiated liposarcomas are associated with abnormalities derived from the q13-15 region of chromosome 12 [1]. Perhaps the best characterized genetic association is in myxoid liposarcoma and represents a translocation or sharing of genetic material between chromosomes 12 and 16. The result is a gene called TLS-CHOP which is an oncogene, or gene that when expressed can lead to the formation of cancer. This particular translocation and its products are specific for myxoid liposarcoma and therefore are diagnostic of this tumor [16].

While many of the principles governing the evaluation and diagnosis of soft tissue sarcomas certainly apply to liposarcoma, there are features unique for the treatment of anterior mediastinal liposarcoma that deserve special consideration. Particularly important is the tumor proximity to the vital mediastinal organs: heart, aorta, lungs, superior vena cava, phrenic nerves and diaphragm. The current standard of treatment is surgical removal and perioperative adjuvant radiation and chemotherapy [7-9]. Surgical procedures can be intralesional (within the tumor mass, often leaving gross tumor), marginal (through the surrounding fibrous membrane, often leaving microscopic foci of tumor), wide (outside the membrane and compartment, leaving no tumor other than "skip metastases"), and radical (most often involving the entire limb and including the entire compartment in which the tumor was located).
In the anterior mediastinum radical and wide resections are frequently unattainable therefore marginal surgery is acceptable for these lesions. The amount of radiation given to a patient may range from 40 to 60 Gy or more, depending on the extent of the surgery, the anatomic site, and likelihood of microscopic or macroscopic retention of diseased tissue [7]. Local control rates of 85-90\% have been achieved with combination therapy of surgery and radiation [8]. The role of chemotherapy in the treatment of liposarcoma is controversial. The principal agents are ifosfamide and doxorubicin, both of which are particularly effective for high-grade tumors [9]. When treated with surgery and perioperative radiation therapy, well-differentiated liposarcomas exhibit a $<10 \%$ local recurrence rate and a virtually $0 \%$ rate of metastasis. In contrast, pleomorphic liposarcomas recur in about $1 / 3$ of cases and spread in about $40 \%$. Five and ten year survival rates for patients with liposarcomas have been reported as $100 \%$ and $87 \%$ for well-differentiated, $88 \%$ and $76 \%$ for myxoid variants and $56 \%$ and $39 \%$ in the pleomorphic subtype $[8,15]$. In some patients because of extensive disease, poor clinical status, and comorbidities, despite a favorable pathological diagnosis, no complete curative treatment can be offered. In our patient, we chose to perform marginal resection to spare the phrenic nerves in an attempt to preserve patient's already compromised respiratory function. Leaving behind microscopic tumor tissue increases the chances of recurrence.

\section{Conclusion}

We reported a case with a large mediastinal mass. Our patient had many of the radiological signs for higher grade malignancy: enormous size, multinodularity with thick calcified septation, but his final histocytological diagnosis was well-differentiated liposarcoma, which has a more benign clinical course. After surgical removal, the recurrence for well-differentiated mediastinal liposarcomas in the mediastinum is unknown; therefore, close follow-up is mandatory.

\section{Consent}

Written informed consent was obtained from the patient for publication of this case report. A copy of written consent is available for review by the Editors-in Chief of this journal.

\section{Competing interests}

The authors declare that they have no competing interests.

\section{Authors' contributions}

SF and KD wrote the manuscript. KD, CG, DH and RT performed surgery. WK and RT supervised manuscript and entire treatment. All authors read and approved the final manuscript. 


\section{References}

1. Enzinger FM, Weiss SW: Liposarcoma. In Soft Tissue Tumors. 3rd edition. Edited by Enzinger FM, Weiss SW. St. Louis, MO: Mosby; 1995:431-466.

2. Dei Tos AP: Liposarcoma: new entities and evolving concepts. Ann Diagn Pathol 2000, 4:252-266.

3. Peterson JJ, Kransdorf MJ, Bancroft LW, O'Connor MI: Malignant fatty tumors: classification, clinical course, imaging appearance and treatment. Skeletal Radiol 2003, 32:493-503.

4. Arkun R, Memis A, Akalin T, Ustun EE, Sabah D, Kandiloglu G: Liposarcoma of soft tissue: MRI findings with pathologic correlation. Skeletal Radiol 1997, 26:167-172.

5. Jung Jl, Kim H, Kang SW, Park SH: Radiological findings in myxoid liposarcoma of the anterior mediastinum. Br J Radiol 1998, 71:975-976.

6. Kransdorf MJ, Bancroft LW, Peterson JJ, Murphey MD, Foster WC, Temple $\mathrm{HT}$ : Imaging of fatty tumors: distinction of lipoma and well-differentiated liposarcoma. Radiology 2002, 224:99-104.

7. Leibel SA, Tranbaugh RF, Wara WM, Beckstead JH, Bovill EG, Phillips TL: Soft tissue sarcomas of the extremities: survival and patterns of failure with conservative surgery and postoperative irradiation compared to surgery alone. Cancer 1982, 50:1076-1083.

8. Zagars GK, Goswitz MS, Pollack A: Liposarcoma: outcome and prognostic factors following conservation surgery and radiation therapy. Int I Radiat Oncol Biol Phys 1996, 36:311-319.

9. Picci P: Adjuvant chemotherapy for extremity soft-tissue sarcomas in adults. Curr Oncol Rep 2000, 2:502-507.

10. Burt M, Ihde JK, Hajdu SI, Smith JW, Bains MS, Downey R, Martini N, Rusch WW, Ginsberg RJ: Primary sarcomas of the mediastinum: results of therapy. J Thorac Cardiovasc Surg 1998, 115:671-680.

11. Ohta Y, Murata T, Tamura M, Sato H, Kurumaya H, Katayanagi K: Surgical resection of recurrent bilateral mediastinal liposarcoma through the clamshell approach. Ann Thorac Surg 2004, 77:1837-1839.

12. Attal H, Jensen J, Reyes CV: Myxoid liposarcoma of the anterio mediastinum. Diagnosis by fine needle aspiration biopsy. Acta Cytol 1995, 39:511-513.

13. Dei Tos AP, et al: From adipocytic tumours. In World Health Organization Classification of Tumours Pathology and Genetics of Tumours of Soft Tissue and Bone. 3rd edition. Edited by Fletcher CDM, Unni KK, Mertens F. Lyon: IARC Press; 2002:35-46.

14. Azumi N, Curtis J, Kempson RL, Hendrickson MR: Atypical and malignant neoplasms showing lipomatous differentiation. A study of 111 cases. Am J Surg Pathol 1987, 11:161-183.

15. Evans HL: Atypical lipomatous tumor, its variants, and its combined forms: a study of 61 cases, with a minimum follow-up of 10 years. Am J Surg Pathol 2007, 31:1-14.

16. Aman $P$, Ron $D$, Mandahl $N$, Fioretos $T$, Heim $S$, Arheden $K$, Willén $H$, Rydholm A, Mitelman F: Rearrangement of the transcription factor gene CHOP in myxoid liposarcomas with $\mathrm{t}(12 ; 16)(\mathrm{q} 13 ; \mathrm{p} 11)$. Genes Chromosomes Cancer 1992, 5:278-285.

\section{Submit your next manuscript to BioMed Central and take full advantage of:}

- Convenient online submission

- Thorough peer review

- No space constraints or color figure charges

- Immediate publication on acceptance

- Inclusion in PubMed, CAS, Scopus and Google Scholar

- Research which is freely available for redistribution 